Pathologe $2015 \cdot 36: 98$

DOI 10.1007/s00292-014-2070-4

(c) Springer-Verlag Berlin Heidelberg 2015

\title{
Dank an die Gutachter von Der Pathologe
}

Der Pathologe ist seit Jahren eine feste Größe in der deutschsprachigen Pathologie. Unter dem Dach der Fachgesellschaften und des Bundesverbandes und unter der Leitung des federführenden Schriftleiters Prof. Dr. K.W. Schmid, Essen, ist dies wesentlich den zahlreichen engagierten Autorinnen und Autoren sowie Ihnen, liebe Leserinnen und Leser, zu verdanken. Maßgeblich für die Qualität und Objektivität der Beiträge sind auch die qualifizierten Gutachter, die im Rahmen des PeerReview-Prozesses nicht nur eingereichte Manuskripte inhaltlich-wissenschaftlich prüfen, sondern auch Empfehlungen zur konkreten Verbesserung äußern. Den hier namentlich aufgeführten Gutachtern und denen, die im Hintergrund bei der Begutachtung mitgewirkt haben, möchten die Schriftleitung von Der Pathologe und die Redaktion für ihre Arbeit im Jahr 2014 herzlich danken.

Dr. Christian August, Münster

Prof. Dr. Hideo Baba, Essen

Prof. Dr. Agnes Bankfalvi, Essen

Prof. Dr. Gustavo Baretton, Dresden

Prof. Dr. Erhard Bierhoff, Bonn

Prof. Dr. Hendrik Bläker, Berlin

Dr. Thomas Brenn, Edinburgh

Prof. Dr. Lukas Bubendorf, Basel

Dr. Henning Feist, Flensburg

Prof. Dr. Falko Fend, Tübingen

Prof. Dr. Ludger Fink, Giessen

Prof. Dr. Hans-Peter Fischer, Bonn

Prof. Dr. Annette Fisseler-

Eckhoff, Wiesbaden

Prof. Dr. Stefan Gattenlöhner, Giessen

Dr. Klaus Griewank, Essen

Prof. Dr. Arndt Hartmann, Erlangen

PD Dr. Kathrin Hauptmann, Berlin

Prof. Dr. Ferdinand Hofstädter, Regensburg

Prof. Dr. Gernot Jundt, Basel

Prof. Dr. Ruth Knüchel-Clarke, Aachen

PD Dr. Andreas Kreft, Mainz

Prof. Dr. Cornelius Kuhnen, Münster
Dr. Florian Laenger, Hannover

Dr. Cord Langner, Graz

Prof. Dr. Sigurd Lax, Graz

Prof. Dr. Volker Loy, Berlin

Prof. Dr. Jutta Lüttges, Hamburg

Prof. Dr. Christian Mawrin, Magdeburg

Prof. Dr. Doris Mayr, München

Prof. Dr. Peter Meister, München

Prof. Dr. Thomas Mentzel, Friedrichshafen

Prof. Dr. Gregor Mikuz, Innsbruck

Prof. Dr. Holger Moch, Zürich

Prof. Dr. Annette Müller, Bonn

Prof. Dr. Klaus-Michael Müller, Münster

Prof. Dr. Felix Offner, Feldkirch

Prof. Dr. German Ott, Stuttgart

Prof. Dr. Iver Petersen, Jena

Dr. Henning Reis, Essen

Dr. Anna-Carinna Reis, Essen

Prof. Dr. Andreas Rosenwald, Würzburg

Prof. Dr. Christoph Röcken, Kiel

Prof. Dr. Thomas Rüdiger, Karlsruhe

Dr. Arno Rütten, Friedrichshafen

Prof. Dr. Wolfgang Saeger, Hamburg
Prof. Dr. Mario Sarbia, München

Prof. Dr. Jörg Schaller, Duisburg

Prof. Dr. Peter Schirmacher, Heidelberg

Prof. Dr. Klaus Schmitz, Recklinghausen

Prof. Dr. Philipp Schnabel, Heidelberg

Prof. Dr. Bence Sipos, Tübingen

PD Dr. Annette Staebler, Tübingen

Prof. Dr. Thomas Stallmach, Zürich

Prof. Dr. Arnulf Stenzl, Tübingen

Prof. Dr. Stephan Störkel, Wuppertal

Prof. Dr. Martin Susani, Wien

Prof. Dr. Andrea Tannapfel, Bochum

Dr. Coya Tapia, Bern

Prof. Dr. Dirk Theegarten, Essen

Prof. Dr. Eva Wardelmann, Münster

Dr. Martin Tötsch, Graz

PD Dr. Michael Vieth, Bayreuth

Prof. Dr. Wilko Weichert, Heidelberg

Prof. Dr. Martin Werner, Freiburg

Prof. Dr. Christian Wittekind, Leipzig

Dr. Katharina Woelke, Berlin

PD Dr. Jeremias Wohlschlaeger, Essen

PD Dr. Jozef Zustin, Münster 